\title{
Perirenal soft tissue infiltration from immunoglobulin G4-related disease
}

\author{
Po-Ting Chen MD, Ko-Ping Chang MD, Kao-Lang Liu MD
}

- Cite as: CMAJ 2018 July 3;190:E801. doi: 10.1503/cmaj.180264

A 62-year-old man presented to our hospital after experiencing two months of diffuse, dull abdominal pain, with scrotal swelling and left epididymitis. Laboratory data showed an increased level of immunoglobulin G4 (IgG4; 3.43 [normal range: 0.032.01] $\mathrm{g} / \mathrm{L}$ ), but were negative for the other autoimmune markers. We noted acute kidney injury with an elevated serum creatinine level (176.8 [normal range: 53-115] $\mu \mathrm{mol} / \mathrm{L}$ ) and reduced urine output. Abdominal computed tomography (CT) showed soft tissue infiltration within the surrounding bilateral perirenal spaces (Figure 1A).

We performed a sonography-guided soft tissue biopsy (supplemental Figure 1A in Appendix 1, available at www.cmaj.ca/ lookup/suppl/doi:10.1503/cmaj.180264/-/DC1). Histological examination showed up to 35 IgG4-positive plasma cells per high-power field (supplemental Figure 1B in Appendix 1). We made a diagnosis of IgG4-related disease. After beginning treatment with a corticosteroid (methylprednisolone, $30 \mathrm{mg} / \mathrm{d}$ ), the patient's urine output increased and serum creatinine levels decreased to $88.4 \mu \mathrm{mol} / \mathrm{L}$ in one week. The follow-up sonography and CT scan showed improvement of the epididymitis and perirenal soft tissue infiltration (Figure 1B).

Immunoglobulin G4 disease is an inflammatory condition that can involve single or multiple organs. Elevation of the level of serum IgG4 should raise the suspicion for this diagnosis, but is not pathognomonic. The definite diagnosis can be made based on imaging, serology (serum IgG4 $>1.35 \mathrm{~g} / \mathrm{L}$ ) and histology (IgG4+ plasma cells $>10 /$ HPF or IgG4:IgG $>40 \%){ }^{1}$

IgG4-related kidney disease usually presents as bilateral multiple renal nodules. ${ }^{2}$ The presentation of perirenal soft tissue infiltration is uncommon, but should provoke further histopathologic work-up. The key histologic findings are lymphoplasmacytic infiltration with increased IgG4-positive plasma cells, and storiform fibrosis. A wide range of manifestations of tubulointerstitial nephritis, membranous glomerulonephritis and chronic sclerosing pyelitis can contribute to renal insufficiency. ${ }^{2,3}$

Immunoglobulin G4-related kidney disease usually responds well to treatment with corticosteroids. The improvement of epi- didymitis in our case is consistent with multiorgan involvement of IgG4-related disease. Even though there is no conclusive treatment, international consensus suggests giving induction therapy (prednisolone $40 \mathrm{mg} / \mathrm{d}$ ) with tapering and aiming to stop treatment in three to six months. ${ }^{1}$ If treated inadequately, IgG4related disease may cause renal dysfunction and progress to irreversible renal damage. ${ }^{3}$

\section{References}

1. Haldar D, Cockwell P, Richter AG, et al. An overview of the diagnosis and management of immunoglobulin G4-related disease. CMAJ 2016;188:953-61.

2. Seo N, Kim JH, Byun JH, et al. Immunoglobulin G4-related kidney disease: a comprehensive pictorial review of the imaging spectrum, mimickers, and clinicopathological characteristics. Korean J Radiol 2015;16:1056-67.

3. Saeki T, Kawano M, Mizushima I, et al. The clinical course of patients with IgG4related kidney disease. Kidney Int 2013;84:826-33.

\section{Competing interests: None declared.}

This article has been peer reviewed.

The authors have obtained patient consent.

Affiliations: Departments of Medical Imaging (Chen, Liu) and Pathology (Chang), National Taiwan University Hospital and National Taiwan University College of Medicine, Taipei, Taiwan

Correspondence to: Kao-Lang Liu, Ikl@ntu.edu.tw 